\title{
Excitation Migration along Oligophenylenevinylene-Based Chiral Stacks: Delocalization Effects on Transport Dynamics
}

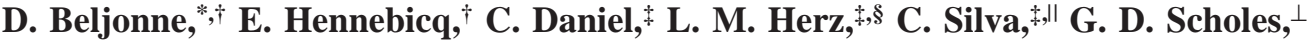 \\ F. J. M. Hoeben, ${ }^{\#}$ P. Jonkheijm, ${ }^{\#}$ A. P. H. J. Schenning, ${ }^{\#}$ S. C. J. Meskers, ${ }^{\#}$ R. T. Phillips, \\ R. H. Friend, and E. W. Meijer ${ }^{\#}$ \\ Laboratory of Chemistry of Novel Materials, University of Mons-Hainaut, Place du Parc 20, 7000 Mons, \\ Belgium, Cavendish Laboratory, University of Cambridge, Madingley Road, Cambridge CB3 OHE, United \\ Kingdom, Clarendon Laboratory, Oxford University, Parks Road, Oxford OX1 3PU, United Kingdom, Lash \\ Miller, Chemical Laboratories, University of Toronto, 80 Saint George Street, Toronto, Ontario M5S 3H6, \\ Canada, and Laboratory of Macromolecular and Organic Chemistry, Eindhoven University of Technology, \\ P. O. Box 513, 5600 MB Eindhoven, The Netherlands
}

Received: February 15, 2005; In Final Form: April 11, 2005

\begin{abstract}
Atomistic models based on quantum-chemical calculations are combined with time-resolved spectroscopic investigations to explore the migration of electronic excitations along oligophenylenevinylene-based chiral stacks. It is found that the usual Pauli master equation (PME) approach relying on uncoherent transport between individual chromophores underestimates the excitation diffusion dynamics, monitored here by the time decay of the transient polarization anisotropy. A better agreement to experiment is achieved when accounting for excitation delocalization among acceptor molecules, as implemented in a modified version of the PME model. The same models are applied to study light harvesting and trapping in guest-host systems built from oligomers of different lengths.
\end{abstract}

\section{Introduction}

Organic $\pi$-conjugated systems are attractive materials to be used as active components in optoelectronic devices ranging in size from molecular to macroscopic dimensions. At the device scale, the demonstration that conjugated polymers (CPs) can be successfully exploited in light-emitting diodes,${ }^{1-3}$ field-effect transistors, ${ }^{4}$ or photodiodes ${ }^{5,6}$ has led to the emergence of a new area in electronics, referred to as "plastic electronics".

The holy grail in plastic electronics is to achieve control of the morphology at the mesoscopic level, which is a formidable task as a result of the inherent disorder present in most CPs. The origin for such disorder is twofold. First, CP chains should be viewed as a superposition of chromophores with different conjugation lengths and hence excitation energies rather than perfect infinite one-dimensional objects. The causes for this are found to be both intrinsic, i.e., self-localization due to coupling to vibrations, and extrinsic, i.e., the presence of structural or chemical defects. ${ }^{7,8}$ Second, on top of this energetic disorder, most conjugated polymers are characterized by a relatively large polydispersity, i.e., the presence of a distribution of physical lengths, leading to the formation of unstructured amorphous films.

As a result, the photophysical properties of CPs are mostly dominated by the way the conjugated chains pack in the solid

* Author to whom correspondence should be addressed. E-mail: David@ averell.umh.ac.be.

University of Mons-Hainaut.

$\div$ University of Cambridge.

$\S$ Oxford University.

" Present address: 'Département de Physique, Université de Montréal,

C. P. 6128, succ. Centre-ville, Montréal, Québec H3C 3J7, Canada.

$\perp$ University of Toronto.

\# Eindhoven University of Technology. state rather than by the single-chain electronic structural properties determined by their detailed chemical structures. That morphology is central to the working principles of polymerbased devices can be easily appreciated in the case of the polymer blends used as active layers in solar cells. In such a case, it is indeed of the utmost importance to achieve phase segregation between the electron-rich and the electron-poor polymer chains, leading to continuous phases for effective hole and electron transport. ${ }^{6}$ As the singlet exciton diffusion range is only on the order of $20 \mathrm{~nm}$, such phase separation should develop on the mesoscopic scale to ensure efficient collection of the photogenerated excitations at the interfacial regions (where dissociation into charges can take place).

Clearly, there is an interest for materials that would combine the ease of processing of conjugated polymers (that are usually spin-coated or cast from solutions) with the reduced energetic and structural disorder of well-defined molecules. One possible strategy in that direction is to exploit the self-organization of electroactive molecules as induced by secondary interactions such as hydrogen bonding to build stable supramolecular structures from solution. This strategy has been exploited in designing substituted chiral oligophenylenevinylene (OPV) molecules that pair via hydrogen bonds and stack to form helical aggregates in low-temperature solutions, Figure $1 .{ }^{9}$ Such welldefined supramolecular architectures, besides being of potential interest for applications in electronics and photonics, are very attractive model systems to explore important photophysical processes such as charge or electronic excitation transport.

Resonant energy transfer (RET), which is the focus of this work, is a ubiquitous process involved in the working mechanism of both biological (e.g., photosynthesis) and artificial antennae. In reference to the example of the solar cells above, the harvesting of singlet excitations at the dissociation zones 
(a)

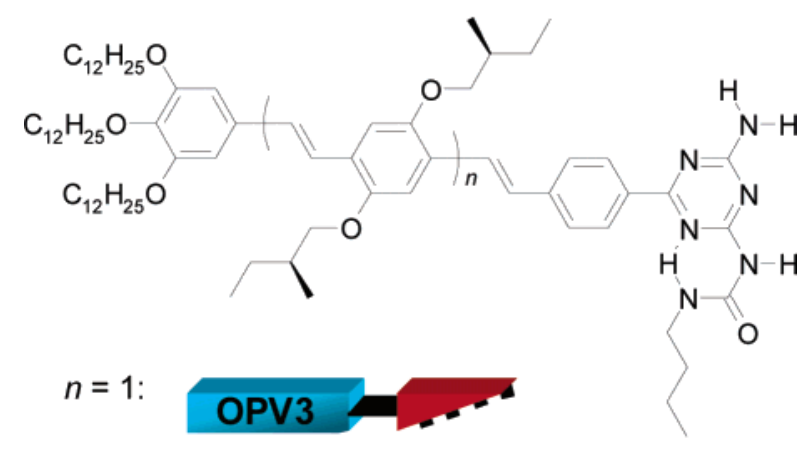

$n=2$ :

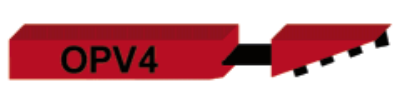

(b)

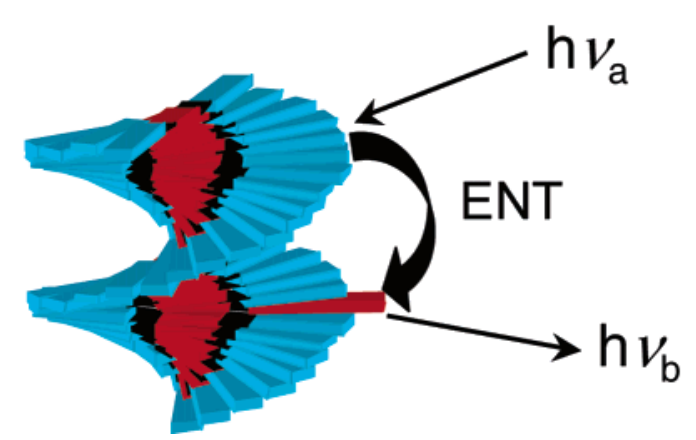

Figure 1. (a) Chemical structure of the OPV derivatives and (b) schematic representation of energy transfer along a mixed OPV3-OPV4 stack.

of polymer blends is believed to be driven by a sequence of RET events taking place within the density of states formed by the distribution of chromophore excitation energies. ${ }^{10,11}$ We have recently applied time-resolved spectroscopy to study the dynamics of excitation motion in OPV chiral stacks doped with acceptor molecules and ascertained the existence of ultrafast energy transfer pathways along the stacks. ${ }^{12}$ Here, we present in-depth models of the energy migration taking place in both pure OPV hosts and in guest-host OPV systems (see chemical structures in Figure 1) based on quantum-chemical calculations in comparison with transient photoluminescence (PL) measurements and polarization anisotropy data obtained by femtosecond transient absorption spectroscopy. We find that uncoherent transport models relying on donors and acceptors localized on individual chromophores underestimate the energy motion dynamics, which are better described when accounting for electronic delocalization among acceptor molecules in a modified master equation formalism.

\section{Experimental Section}

Polarization anisotropy measurements were made by means of femtosecond transient absorption spectroscopy. A Ti:sapphire oscillator (KMLabs) produced 15-fs pulses that were amplified using a chirped-pulse-amplification scheme at a repetition rate of $1 \mathrm{kHz}$. The amplifier was a home-built multipass Ti:sapphire amplifier (pumped by the 9.5-W, 527-nm output from a SpectraPhysics Evolution $\mathrm{X}$ laser). The resulting pulses had pulse widths of $70 \mathrm{fs}$, pulse energies of $900 \mu \mathrm{J}$, and were centered at a wavelength of $780 \mathrm{~nm}$.

The sample was excited with 3.1-eV pump pulses produced by frequency doubling the laser fundamental in a $0.5-\mathrm{mm}$ path length $\beta$-barium borate (BBO) crystal. The pump power was varied with calibrated neutral density filters, and the beam was mechanically chopped at $500 \mathrm{~Hz}$. The transient absorption of the sample was probed with a single-filament white-light continuum (approximately from 1.2 to $3.0 \mathrm{eV}$ ) produced by focusing a fraction of the fundamental into a sapphire window. Probe pulses were delayed with respect to pump pulses with a computer-controlled optical delay. The beams were linearly polarized with a relative angle of $0^{\circ}$ or $90^{\circ}$ in parallel and perpendicular configurations, respectively. The probe and the pump beams were focused on the sample cell to $\sim 50$ and $\sim 150 \mu \mathrm{m}$ spots, respectively, with an angle between beams of approximatively $20^{\circ}$. The probe beam and a reference beam (not transmitted through the sample) were dispersed in a $0.25-\mathrm{m}$ spectrometer and detected with a pair of avalanche photodiodes. The pump-induced variations in transmission $(\Delta T / T)$ were extracted using a real-time algorithm that digitized each probe laser shot, applied statistical criteria to reject shots where the integrated intensity varied outside a predetermined deviation from the mean intensity, and averaged the data over several thousand shots per time delay point. The instrument response function had a full width at half-maximum (fwhm) of less than $120 \mathrm{fs}$. The anisotropy was defined as $\left(\left(\Delta T_{\|}-\Delta T_{\perp}\right) /\right.$ $\left.\left(\Delta T_{\|}+2 \Delta T_{\perp}\right)\right)$.

To permit the investigation of excitation transfer in mixed OPV stacks, a blend of OPV3 and OPV4 in dodecane solution $\left(1.85 \times 10^{-4} \mathrm{M}\right.$ containing $2.6 \%$ of OPV4) was added to a Spectrosil cuvette mounted in a temperature-controlled holder. Care was taken to heat the blend initially above the transition temperature to ensure that mixed stacks formed upon subsequent cooling. ${ }^{12}$ The photoluminescence emitted after excitation was measured as a function of time using the up-conversion (UC) technique providing a resolution of $350 \mathrm{fs}$. The frequencydoubled output from a mode-locked Ti:sapphire laser was used to excite the sample with 200-fs pulses at an energy of $3.06 \mathrm{eV}$ and an excitation intensity of $0.7 \mathrm{~mW}$. Photoluminescence emerging at a right angle to the exciting beam was collected using a pair of off-axis parabolic mirrors and up-converted in a BBO crystal using the fundamental laser beam at $1.53 \mathrm{eV}$ as a gate. Sum-frequency photons were dispersed in a monochromator and detected by a cooled photomultiplier tube using photon counting techniques. The UC data shown were taken for collinear polarization of the exciting and the detected light. To provide a measure of the pure transfer dynamics from host to guest molecules, the photoluminescence decay from the blend was taken in the spectral region where the guest emits $(557 \mathrm{~nm})$ and divided by the luminescence decay measured for undoped stacks of OPV.

\section{Resonant Energy Transfer in OPV Stacks: Master Equation Formalisms}

Within the Markovian approximation (memory effects between donors and acceptors are neglected), the dynamics of energy migration within a multichromophoric system obeys the following set of PMEs ${ }^{13,14}$ for the time-dependent occupation probability on chromophore $m, P_{m}(t)$

$$
\underset{\partial t}{\frac{\partial}{\mathrm{m}}} P_{n}(t)=\sum_{n \neq m}\left(k_{m n} P_{m}(t)-k_{n m} P_{n}(t)+P_{m} \tau_{m}^{-1}\right)
$$

where $k_{m n}$ is the rate for excitation energy transfer from site $m$ to site $n$ and $\tau_{m}$ is the lifetime for the excitation on site $m$. The first term on the right-hand side of eq 1 describes the decay with a time of $P_{m}(t)$ as a result of exciton migration from site 
$m$ to all other sites; the second term is associated with the reverse hopping to site $m$ from all sites $n \neq m$; the last term accounts for the natural exciton lifetime in the absence of any transfer process (this term is neglected in the following as we are interested in time scales shorter than the excited-state lifetimes).

Assuming both fast thermalization in the excited state (geometric relaxation occurs prior to hopping, i.e., "hot" energy transfer events taking place during geometric relaxation phenomena on the excited donor are not accounted for) and no coupling between acceptors (no coherent effects), $k_{m n}$ can be derived from time-dependent perturbation theory and the Fermi golden rule $(\mathrm{FGR})^{15,16}$

$$
k_{m n}=\frac{2 \pi}{\hbar} V_{m n}^{2} J_{m n}
$$

where $V_{m n}$ is the electronic matrix element for energy transfer, which can be viewed as a measure of the electronic interaction between excitations localized over sites $m$ and $n ; J_{m n}$ denotes the spectral overlap between the (donor) emission spectrum of $m$ and the (acceptor) absorption spectrum of $n$ and ensures energy conservation. Within the regime described by eqs 1 and 2 , energy migration thus takes place through sequential incoherent hopping steps between fully localized chromophores.

Previous experimental investigations of the photophysical properties of OPV dodecane solutions have highlighted the following. In the molecularly dissolved state at "high" temperatures (i.e., $T$ larger than the concentration-dependent transition temperature $T_{\mathrm{c}}{ }^{9}$ ), a large Stokes shift (of about $0.5 \mathrm{eV}$ ), unresolved within the subpicosecond resolution of the experimental apparatus used, is observed. ${ }^{17}$ Such a Stokes shift primarily corresponds to the energy gain associated with a change in conformation toward a more planar structure in the excited state. In the helical aggregates at "low" temperatures ( $T<T_{\mathrm{c}}$ ), a more limited red shift of the emission then develops within the first few tens of picoseconds, which is ascribed to downhill migration of the excitations toward lower-energy sites. These low-energy species could correspond to either molecules with slightly flatter backbones or molecules lying in a different environment.

The steady-state PL spectra are dominated by the emission from molecular aggregates (associated, e.g., with slight changes in the interdistance packing or in the relative orientations of the molecules), as suggested by the reduced emission quantum yields measured for the stacks as compared to the isolated molecules ${ }^{17,18}$ (as intermolecular interactions lead to an increased radiative lifetime in the chiral aggregates) and the circularly polarized emission signal. ${ }^{19}$ Electronic coupling between the OPV molecules in their ground-state geometries ("acceptors") show up very clearly in the circular dichroism (CD) spectra recorded for the helical stacks (these indicate a strong bisignated Cotton effect at the $\pi-\pi^{*}$ absorption associated with delocalized acceptor excited states). ${ }^{17}$ Switching on the intermolecular interactions upon cooling the dodecane solutions below $T_{\mathrm{c}}$ is also accompanied by the emergence of a low-energy shoulder in the absorption.

According to the description above, it seems reasonable to sort the successive steps in the energy transfer process according to the following time line. Light absorption first prepares coherent (delocalized) excited species, as indicated by the CD data $^{17}$ (although the amount of delocalization is probably limited by energetic disorder). Here we assume that fast ( $\ll 1 \mathrm{ps})$ decoherence driven by structural (mostly conformational) relaxation then yields excitations localized on individual OPV molecules. These migrate within the density of states created by the distribution of chromophore energies (inhomogeneous broadening) to reach low-energy sites (presumably more planar conformers or aggregates) where they decay back to the groundstate giving rise to light emission.

As in the time window considered here ( $>1$ ps) selflocalization leads to decoupled energy donor and acceptor molecules, excitation transport can still be reasonably accounted for in a Markovian picture. In the limiting case where both donor and acceptor states are fully localized on single chromophore units, the simulation of the long-range RET dynamics proceeds through eqs 1 and 2. To include excitation delocalization in the acceptor manifold (self-localization on the donor is still assumed) while keeping the simplicity and insightfulness of the Markovian approach, we have developed a modified version of the PME formulation (hereafter referred to as PME-d, where "d" stands for "delocalized") where the $k_{m n}$ hopping rates between donor $m$ and acceptor $n$ are expanded in terms of acceptor effective states $\alpha$

$$
k_{m n}=\sum_{\alpha} k_{m \alpha} \mathrm{C}_{m \alpha}^{2}
$$

with

$$
k_{m \alpha}=\frac{\hbar}{2 \pi} V_{\mathrm{m} \alpha}^{2} J_{\mathrm{m} \alpha}=\frac{2 \pi}{\hbar}\left(\sum_{p \neq m} V_{\mathrm{mp}} \mathrm{C}_{\mathrm{p} \alpha}\right)^{2} J_{\mathrm{m} \alpha}
$$

where $C_{n \alpha}$ is the contribution from site $n$ to the acceptor effective state $\alpha$, as obtained by diagonalizing the following purely electronic excitonic Hamiltonian for the supramolecular aggregate formed by all sites except $m$

$$
H=\sum_{n \neq m} \sum_{k \neq m}|n\rangle\langle k|\left(H_{\mathrm{nn}} \delta_{n k}+V_{\mathrm{nk}}\right)
$$

with $H_{n n}$ the excitation energy on site $n$.

Equation 3 assumes fast thermalization of the donor after excitation, with a probability for the excitation to self-localize on chromophore $n$ given by the corresponding squared coefficient in the wave function, $C_{n \alpha} . V_{m \alpha}$ and $J_{m \alpha}$ in eq 4 are the electronic coupling and spectral overlap between the donor state localized on $m$ and the effective "delocalized" acceptor state $\alpha$, respectively ( $V_{m \alpha}$ can be readily expressed in terms of the coupling matrix elements between the localized sites as indicated in eq 4 , which retains both the squared wave function coefficients and the cross terms). It is readily seen that, in the case of complete localization of the acceptor wave functions (corresponding to vanishing interchromophore couplings), the summation in the expression of the effective coupling $V_{m \alpha}$ in eq 4 reduces to a single term (all coefficients $C_{p \alpha}$ are zero except on site $n$ where $C_{n \alpha}=1$ ) and eq 2 is recovered. For nonzero coupling matrix elements, the acceptor wave functions can delocalize over neighbor accepting chromophores, whose contributions to the transfer rate from donor $m$ are weighted by the associated coefficients in the effective excited-state wave functions. Thus, in this approach, coherent effects among acceptors are retained while the initial donor state is assumed to be in thermal equilibrium after complete vibrational relaxation. Equations 3 and 4 combined with eq 1 hence provide a simple yet realistic way of gauging the impact on the energy transfer dynamics of the formation of excitonlike acceptor states, which is completely neglected in the conventional PME approach.

Energy transfer promoted by Coulomb interactions, which are the only contributions retained in the Förster mechanism, 
is a long-range process whose efficiency is directly related to the dipole strength of the donor and acceptor electronic transitions. In the original Förster model, $V_{m n}$ is further simplified by truncating after the dipolar term the interactions in a multipole expansion with respect to the $m n$ center-to-center distance. ${ }^{20}$ Such an approach averages away the shapes of the donor and acceptor wave functions and should only be applied when the size of the interacting molecules is small with respect to the intermolecular separation. To account for the detailed chemical structure and topology of the interacting chromophores, we have adopted an "improved" Förster formalism, wherein the total electronic coupling is expressed as a sum over pairwise interactions between atomic transition charges ${ }^{21-24}$

$$
V_{n m}=\frac{1}{4 \pi \epsilon_{0}} \sum_{i}^{m} \sum_{j}^{n} \frac{\rho_{m}(i) \rho_{n}(j)}{r_{i j}}
$$

where the summation runs over all sites $i(j)$ on molecule $m(n)$ and $r_{i j}$ is the distance between atomic centers $i$ and $j ; \rho_{m}(i)$ $\left(\rho_{n}(j)\right)$ denotes the atomic transition density on site $i(j)$ for the lowest optical excitation on molecule $m(n)$. The transition densities provide a local map of the interacting transition dipole moments and can be obtained from quantum-chemical calculations on the isolated chromophores (vide infra).

The spectral overlap factors, $J_{m n}$ in eq 2 , have been estimated from the normalized donor emission and acceptor absorption spectra, as simulated within the Franck-Condon approximation and the displaced harmonic oscillator model. ${ }^{25}$ Within such a model, it is assumed that the electronic ground and excited state of the molecules can be treated as undistorted harmonic oscillators (their potential energy curves are described by parabolas with identical curvatures), which are displaced with respect to one another along some normal vibrational modes. The vibrational progression is then written as a simple product of Boltzmann-weighted Franck-Condon integrals (FCI) over all vibrational modes $i$ that are coupled to the electronic excitation

$$
\prod_{i} \operatorname{FCI}\left(p_{i}, l_{i}\right)^{2} \exp \left(-\frac{\sum_{i} p_{i} h v_{i}}{k T}\right)
$$

with

$$
\operatorname{FCI}\left(p_{i}, l_{i}\right)^{2}=\exp \left(-\frac{S_{i}}{2}\right) \sum_{j=0}^{p_{i}} \frac{(-1)^{j} S_{i}^{j / 2} S_{i}^{\left(l_{i}-p_{i}+j\right) / 2} \sqrt{l_{i} ! p_{i} !}}{j !\left(l_{i}-p_{i}+j\right) !\left(p_{i}-j\right) !}
$$

where $p_{i}$ and $l_{i}$ stand for the vibrational quantum numbers for mode $i$ in the initial (i.e., ground state for absorption and excited state for emission) and final states, respectively; $v_{i}$ denotes the vibrational frequency of mode $i$ and $S_{i}$ the associated HuangRhys factor. $S_{i}$ is a measure of the electron-phonon coupling and corresponds to the ratio of the nuclear reorganization energy along the normal coordinate and the corresponding vibrational energy $h v_{i \cdot}{ }^{26}$ At $0 \mathrm{~K}$, only the fundamental vibrational level is populated in the initial state, and eq 6 reduces to a simple and widely used Poisson distribution. ${ }^{26}$ When $h v_{i}$ is comparable to $k T$ (which is the case for soft modes such as libration modes at room temperature), the redistribution of the population over the vibrational levels in the starting electronic state, taken into account via the Boltzmann factors in eq 7, leads to broadened and shifted spectral lines. ${ }^{27,28}$ As will be shown below, the thermal population of these low-frequency vibrational modes has a strong impact on the spectral overlap factors and as a result the energy migration dynamics.

The optical absorption and emission spectra are obtained by convoluting the calculated Franck-Condon transitions with Gaussian functions of fwhm homogeneous broadening (HB) and by weighting them with the appropriate frequency factors for absorption and spontaneous emission processes (frequency and frequency to the cube for absorption and emission, respectively). The spectral overlaps $J_{m n}$ are then computed numerically from the donor emission and acceptor absorption spectra, after normalization to the unit area on an energy scale. The calculation of the effective overlaps $J_{m \alpha}$ in eq 4 is more difficult to handle, as it requires the characterization of the geometric relaxation taking place in each exciton state $\alpha$ (while our excitonic Hamiltonian in eq 5 is purely electronic); to a first approximation, we have assumed that these are similar to the lattice distortions occurring in single chromophores (hence the same vibrational frequencies and Huang-Rhys factors were used). We recognize that this is a rather crude approximation, e.g., it is likely that the changes in conformation are less pronounced in the stacks than for the dissolved species (a more detailed investigation of the spectroscopic properties of the chiral aggregates is presented elsewhere) ${ }^{29}$ However, it allows for a direct comparison to the PME results and provides a simple hint to the effects of electronic delocalization among acceptors on the dynamics.

From the solutions of eq 1 (obtained through numerical diagonalization of the stochastic matrix involving the transfer rates), ${ }^{30}$ one can derive a series of useful observables, allowing for direct comparison to experimental data. In the following, we will mostly focus on the mean values of the electronic excitation energy, $E^{*}(t)$, squared displacement, $d^{2}(t)$, and emission polarization anisotropy, $r(t)$, defined as

$$
\begin{gathered}
\left\langle E^{*}(t)\right\rangle=\frac{\sum_{n} P_{n}(t) E_{n}^{*}}{\sum_{n} P_{n}(t)} \\
\left\langle d^{2}(t)\right\rangle=\frac{\sum_{n} P_{n}(t) d_{n}^{2}}{\sum_{n} P_{n}(t)} \\
\langle r(t)\rangle=\left\langle\frac{I_{\|}-I_{\perp}}{I_{\|}-2 I_{\perp}}\right\rangle_{(t)=\frac{1}{5}} \frac{\sum_{n} P_{\mathrm{n}}(t)\left\{3 \cos ^{2} \alpha_{n}-1\right)}{\sum_{n} P_{n}(t)}
\end{gathered}
$$

where $E_{n}^{*}=H_{n n}$ is the excitation energy of chromophore $n ; d_{n}$ is the average center-to-center distance between site $n$ and the initially excited molecule; $I_{\|}$and $I_{\perp}$ stand for the parallel and perpendicular components of the light emission with respect to the excitation laser polarization, respectively. Equation 10 was derived assuming cylindrical aggregates representative of the chiral one-dimensional stacks with the chromophore transition dipole moments lying in a plane perpendicular to the stacking direction; $\alpha_{n}$ is then the in-plane angle formed by the transition dipole moment of molecule $n$ and that of the molecule that originally absorbed the photon. $\langle r(t)\rangle$ is predicted to evolve from 0.4 at time $t=0$ to a limiting value of 0.1 in cases of complete 
a)

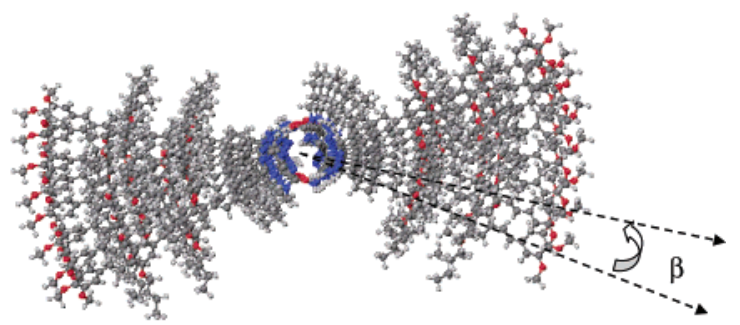

b)

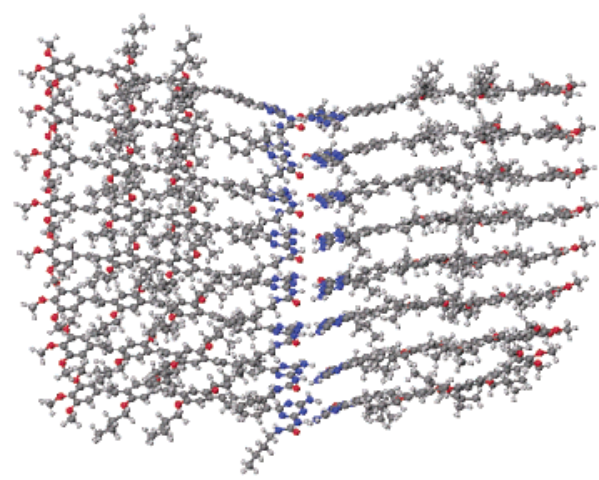

Figure 2. (a) Top and (b) side views of a 16-molecule stack of OPV4, as optimized at the Dreiding force field level. $\beta$ denotes the rotation angle between two consecutive molecules along the stack.

depolarization within the same one-dimensional helical stack (it converges to 0 at $t=\infty$ if the excitation is allowed to jump between distinct randomly oriented molecular stacks). The loss of polarization anisotropy as the excitations migrate along the stack is thus a very useful marker for the excitation motion dynamics.

As quoted above, the Stokes shift observed at $t>1 \mathrm{ps}$ in the chiral stacks can be partly attributed to energy hopping within the density of states (DOS) formed by OPV molecules with slightly different excitation energies. To model such disorder, the site energies were sampled within a Gaussian DOS with fwhm inhomogeneous broadening (IB). The simulations of energy transport were performed on stacks comprising a few hundred chromophores and repeated typically a few thousand times to average over disorder.

\section{Description of the Stacks and Model Parameters}

There is compelling experimental evidence, from $\mathrm{CD}$, absorption, and photoluminescence spectroscopy, that the OPV molecules form helical aggregates in "poor" solvents (e.g., dodecane) at low temperatures. ${ }^{17}$ However, structural information on the stacks, such as, e.g., the helix pitch, is lacking. Hence, to provide some insight into the supramolecular organization, molecular mechanics calculations based on the Dreiding force field (which is particularly suitable for hydrogen-bonded systems ${ }^{31}$ ) were performed on model clusters including 16 OPV4 molecules (i.e., 8 dimers); see Figure 2. All structural parameters were optimized starting from initial configurations that differ by the angle $\beta$ between neighboring superimposed chromophores (chosen to vary from $0^{\circ}$ to $20^{\circ}$ ). The most stable structures were obtained for angles between the main chain axes of the molecules in the range $3-6^{\circ}$. This is likely a lower limit, since these calculations deal with isolated stacks ignoring their environment (hence competitive interactions with the solvent); thus, the close packing of the OPV molecules through reduced misalignment of their main axes is likely overestimated. Simulations of the photophysical properties of the stacks, reported elsewhere, ${ }^{29}$ show that an excellent agreement to experiment is obtained when enforcing angles in the range $6-12^{\circ}$. We have hence performed the simulations of excitation motion for $\beta$ values spanning a range from $3^{\circ}$ to $12^{\circ}$.

The geometric structures of long one-dimensional clusters have been built on the basis of the Dreiding helical structures while refining the intramolecular parameters at the quantumchemical AM1 $1^{32}$ level. In addition, the lengths of the $\mathrm{NH} \cdots \mathrm{O}$ and $\mathrm{NH} \cdots \mathrm{N}$ hydrogen bonds, slightly underestimated by the Dreiding force field, were adjusted according to experimental $\mathrm{X}$-ray data obtained for the tetrafunctionalized hydrogen-bonded linker compound; ${ }^{33}$ the intermolecular separation along the stacks was fixed to $3.75 \AA$ according to the force field calculations.

The AM1-optimized geometries of the chomophores were used as input for excited-state calculations performed at the highly correlated $\mathrm{INDO}^{34} / \mathrm{CCSD}^{35,36}$ level. As an output of those calculations, the atomic transition densities involved in the calculation of the electronic couplings in eq 6 are provided. These are used to estimate the hopping rates between all pairs of molecules by means of either eq 2 for PME or eqs 3 and 4 for PME-d. As an illustration, the INDO/CCSD nearest-neighbor interactions amount to $\sim 460(370) \mathrm{cm}^{-1}$ for hopping between superimposed molecules for a $6^{\circ}\left(12^{\circ}\right)$ rotation and $\sim 70 \mathrm{~cm}^{-1}$ for hopping between chromophores belonging to the same hydrogen-bonded dimer. To a first approximation, the system can be regarded as mainly one-dimensional (with stronger interchromophore couplings along the stack than between twin molecules of the same pair). Yet, hopping through the hydrogen bonds is characterized by a significant electronic matrix element and plays a substantial role in the trapping dynamics in guesthost systems (vide infra).

A proper description of energy migration within disordered systems requires (i) first a calculation of the spectral overlap factors between homogeneously broadened donor emission and acceptor absorption spectra and the solution of the corresponding PME (or PME-d) and (ii) then an average over inhomogeneous broadening by repetition of the simulations for different sets of site energies defined within a given (usually Gaussian) distribution. Hence, the measured absorption and emission spectra in solution, which represent ensemble average quantities, cannot be used as such in the computation of the spectral overlaps and the simulation of the energy transfer dynamics. However, these measurements provide independent information on the spectral shapes and vibronic couplings, which allow testing of the accuracy of the model.

To simulate the optical spectrum, the normal-mode frequencies and displacements entering eq 7 need to be fixed. Quantumchemical calculations on phenylene-based compounds show that optical excitation into the lowest singlet excited state couples to a limited number of vibration modes. ${ }^{27}$ Here, a simple twomode model that captures the main spectral features of the complex absorption and emission spectra has been considered. It includes (i) a high-frequency mode (at $0.17 \mathrm{eV}$ ) representative of the dominant aromatic carbon-carbon stretching and ring breathing vibrational motions and (ii) a low-frequency libration mode (at $0.01 \mathrm{eV}$ ). These indeed mediate the bond-length deformations toward a more quinoidlike structure and concomitant conformation changes toward a more planar backbone when going from the ground state to the excited state in phenylenebased conjugated systems.

As described by Karaburnaliev and Bittner, ${ }^{27}$ coupling to optical excitations of soft modes with high Huang-Rhys factors (typical for floppy phenylene-based compounds) introduces a 


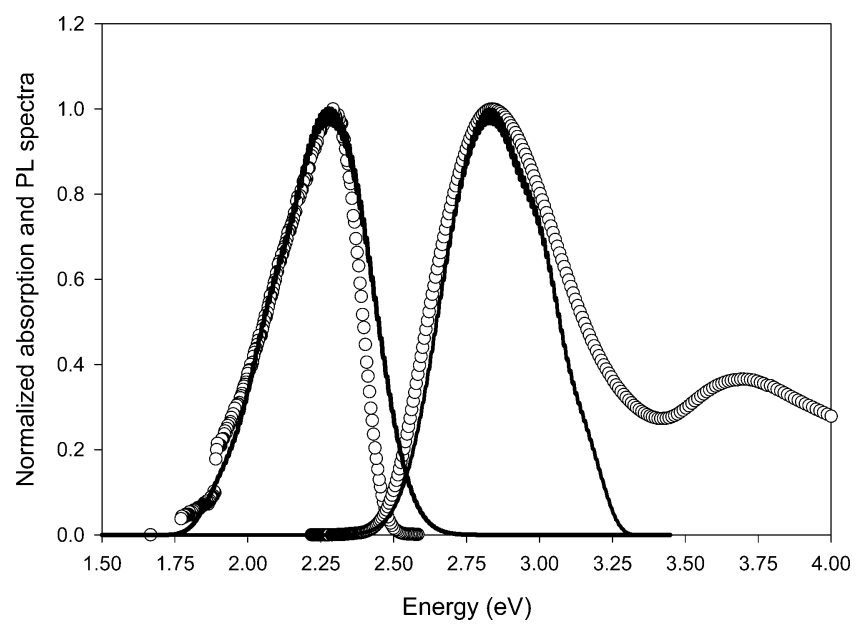

Figure 3. Comparison between the measured (open symbols; dodecane solutions at $90^{\circ} \mathrm{C}$ ) and calculated (solid lines) absorption and emission spectra of OPV4.

Stokes shift between the "apparent" (the low-frequency modes cannot be resolved) absorption and emission 0-0 lines and leads to a temperature-dependent broadening of the vibrational features. As a result of this Stokes shift, the spectral overlap between absorption and emission spectra is significantly reduced and strongly temperature dependent; it actually increases with increasing temperature due to population of higher-lying vibrational levels in the initial electronic state (hence leading to the appearance of an anti-Stokes phonon wing on the blue side in emission and on the red side in absorption).

Here, the low-frequency mode Huang-Rhys factor involved in the calculation of the Franck-Condon overlaps has been adjusted to fit the Stokes shifts measured in OPV4 solutions at room temperature; $S \approx 20$, corresponding to a Stokes shift of $2 S h v \approx 0.4 \mathrm{eV}$ (with $h v=0.01 \mathrm{eV}$ ), was found to lead to the best agreement with experiment. Note that this is higher than the value reported for the libration mode in oligophenylenes $;{ }^{27}$ one should thus regard this vibration mode as an effective mode accounting for all low-frequency vibrations coupled to the excitation (i.e., not only libration but also other soft acoustic modes such as intermolecular modes, etc.). ${ }^{37}$ The Huang-Rhys factor associated with the high-frequency vibrational mode has been fixed to 1.0, a typical value for phenylene-based oligomers.

Disentangling the homogeneous and inhomogeneous broadening contributions is a difficult task. Single-molecule spectroscopic investigations in a ladder-type polyparaphenylene suggest that $\mathrm{HB}$ might be as small as a few millielectronvolts at low temperature, which translates into dephasing times on the order of a few hundreds of femtoseconds. ${ }^{38}$ Here, we have considered a value of $10 \mathrm{meV}$ (fwhm) for the purely electronic transition and its vibronic replica at $0 \mathrm{~K}$, in line with these experimental results. As mentioned above, increasing the temperature leads to a redistribution of the populations in the vibrational sublevels and a consequent broadening of the spectral lines. At room temperature, the total line widths result from the superposition of the homogeneous and inhomogeneous contributions; the latter was set to $100 \mathrm{meV}$ (fwhm) to reproduce the absorption and emission spectra measured at room temperature. We stress that combining these values for HB and IB leads not only to a good fit of the experimental inhomogeneously broadened spectra but also to excitation energy distributions at long time delays obeying detailed balance conditions (vide infra).

The room-temperature simulated absorption and emission spectra of the OPV4 molecule are compared to the corresponding experimental spectra in Figure 3. Taking into account the simplicity of the vibronic model used, the agreement between theory and experiment is very satisfactory. Note that (i) a more quantitative treatment would require the use of different chromophore energy distributions in the ground state and in the excited state, as these are likely characterized by different shapes of their potential energy curves (a feature that partly contributes to the lack of mirror symmetry between absorption and emission); ${ }^{39}$ this is, however, beyond the scope of the current paper. (ii) At the measured solution concentration $\left(10^{-4} \mathrm{M}\right)$, self-absorption cannot be completely excluded, which might explain the faster falloff of the measured response on the highenergy side of the PL compared to that of the calculated spectrum.

To check that the choice of model parameters above provides a physically sound description of the system, we have explored the temperature-dependent excitation transport dynamics at the PME level ( $\beta$ is fixed to $12^{\circ}$ in these calculations). At long time delays, the mean excitation energy as calculated from eq 8 using the populations supplied by solving the master equations should indeed converge toward the thermodynamic limit, $\left\langle E^{*}(\infty)\right\rangle$

$$
\left\langle E^{*}(\infty)\right\rangle=\frac{\sum_{n} E_{n}^{*} \exp \left(-\frac{E_{n}^{*}}{k T}\right)}{\sum_{n} \exp \left(-\frac{E_{n}^{*}}{k T}\right)}
$$

The time-dependent average energy, squared displacement, and polarization anisotropy, reported in Figure 4 for $T$ ranging from 200 to $500 \mathrm{~K}$, call for the following comments (note that these simulations have no experimental counterpart, as the OPV molecules only form chiral aggregates in a narrow temperature range):

(i) At room temperature or above, $\left\langle E^{*}(t)\right\rangle$ converges to the thermodynamic limit in eq 11 within $\sim 400$ ps at $300 \mathrm{~K}$ and $\sim 50$ ps at $500 \mathrm{~K}$; a slower evolution is predicted below room temperature with no indication of convergence to the equilibrium within the first 500 ps at $200 \mathrm{~K}$.

(ii) The loss of polarization anisotropy follows the same trend; it is complete and fast at room temperature and above, indicative of efficient energy migration, while at $200 \mathrm{~K}$ the system seems to be "frozen" owing to slow downhill and uphill hopping rates.

(iii) The faster excitation motion at higher tempeature translates into larger mean-squared displacements, i.e., at $300 \mathrm{~K}$ the excited species explore $\sim 40 \AA$ (root-mean-square (rms) distance) around the initially excited chromophore within the first $100 \mathrm{ps}$ while this distance increases to $\sim 90 \AA$ at $500 \mathrm{~K}$.

The crossover from slow energy transfer at low temperature to fast migration at high temperature results from the temperature-dependent homogeneous line width and spectral overlap. ${ }^{28}$ While HB is small compared to the Stokes shift at low temperature (hence resulting in small spectral overlaps and hopping rates), redistribution of the populations taking place among the vibrational states of the low-frequency mode at high temperature results in an increased (effective) HB, thereby leading to enhanced spectral overlaps and transfer rates.

\section{Excitation Diffusion and Trapping Dynamics}

The model described in section III and validated by comparison of the simulated and measured optical spectra in section IV has been applied to the description of excitation diffusion 
(a)

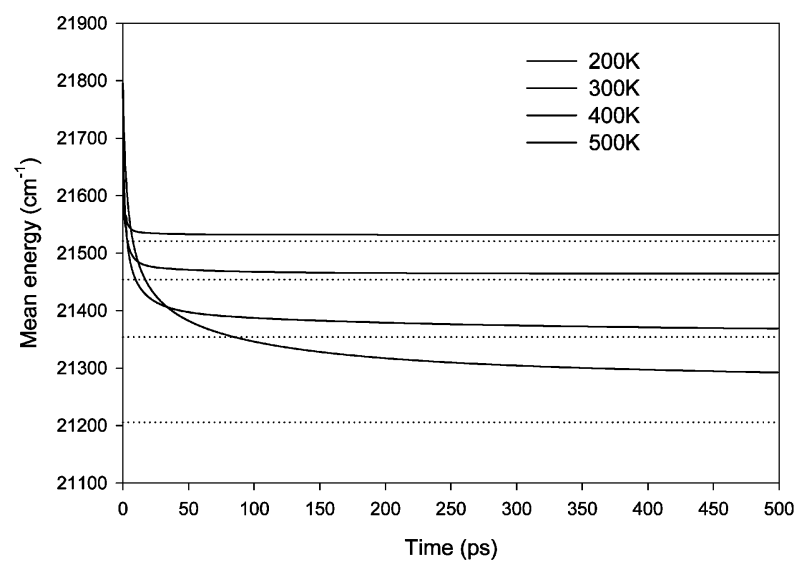

(b)

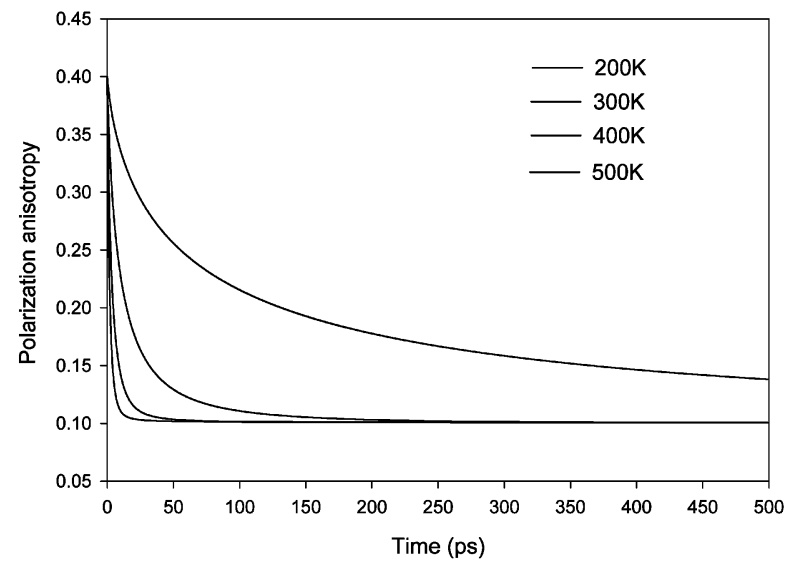

(c)

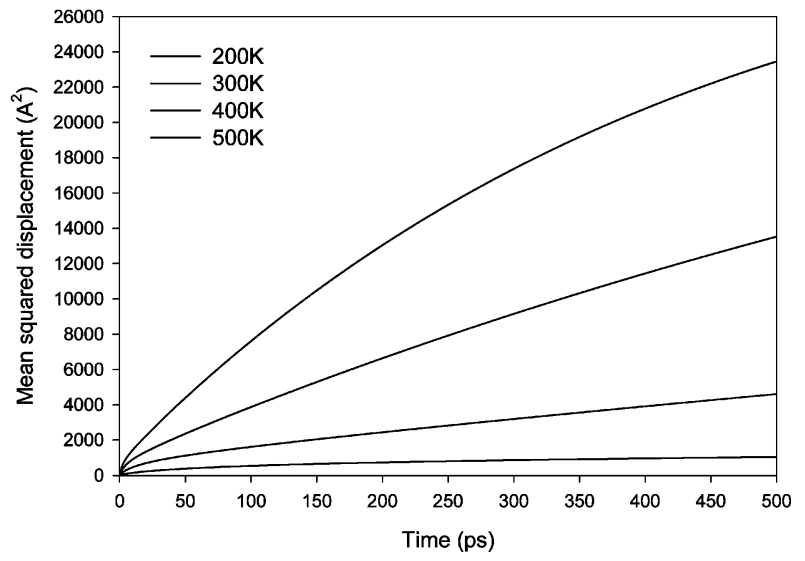

Figure 4. (a) Time-dependent mean excitation energy, (b) emission polarization anisotropy, and (c) squared displacements, as predicted by a PME model for temperatures ranging from 200 to $500 \mathrm{~K}$ and a rotation angle, $\beta$, of $12^{\circ}$.

in OPV4 stacks as well as energy trapping in host-guest systems. To characterize the migration dynamics in the pure OPV4 host material, we have followed the evolution with time of the transient polarization anisotropy, $r(t)$, as obtained by means of both the PME and GPME approaches and assuming a fixed rotation angle, $\beta$, between superimposed OPV molecules (in the range $3-12^{\circ}$; see above). A comparison between the calculated and measured anisotropy decay during the first 70 ps is shown in Figure 5a.

The experimental data (obtained from transient absorption spectroscopy) indicate an ultrafast decay of the polarization anisotropy consistent with rapid motion of the excitations along the stacks. $r(t)$ reaches half its initial value after less than $10 \mathrm{ps}$ and converges to the limiting 0.1 value within $\sim 30$ ps (note that the polarization anisotropy further decreases toward zero for time delays in excess of $1 \mathrm{~ns}$, as a result of "interstack"
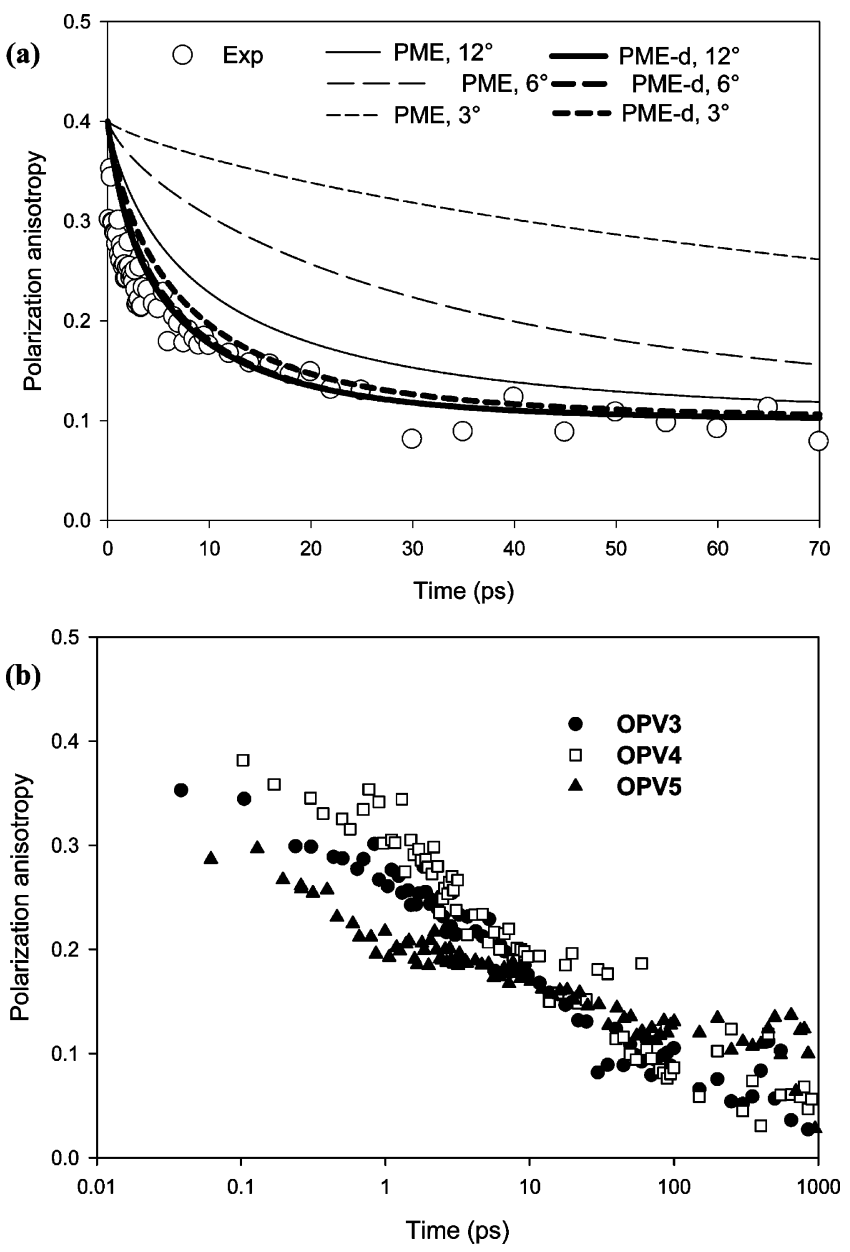

Figure 5. (a) Time-dependent emission polarization anisotropy at the PME and PME-d levels along OPV4 stacks at room temperature and for different values of the rotation angle $\beta$. The experimental values are indicated by open symbols. (b) Measured time decay of the polarization anisotropy in stacks of OPV3, OPV4, and OPV5 molecules.

migration, Figure 5b). The time-dependent anisotropies calculated within the PME approximation show a strong dependence on the $\beta$ value. Although characterized by smaller electronic couplings, larger rotation angles translate into a faster loss of polarization anisotropy, as a result of a more important drop in $r(t)$ after each single hop. Yet, even for $\beta=12^{\circ}$, which is likely an upper limit for the revolution angle around the stacking direction, the PME results lead to a diffusion of the excitations that is too slow compared to experiment. In contrast, the PME-d simulations provide $r(t)$ decay curves almost insensitive to the $\beta$ value and in better agreement with the measured evolution.

To understand the differences between the PME and PME-d results, it is useful to investigate the scaling with intermolecular distances of the computed hopping rates predicted by both formalisms. Such evolutions are shown in Figure 6 for pairwise hopping from the top site of a $12^{\circ}$ stack to all other chromophores. As expected, the hopping rates calculated according to eq 2 and used in the PME model show a strong decrease with the center-to-center separation between the molecules (in the long-distance limit, these scale as the sixth power of the inverse distance; note that the fluctuations are due to changes in the spectral overlaps associated with disorder). In contrast, a much weaker evolution is obtained for the rates computed on the basis of eqs 3 and 4 and the PME-d model. This can be understood from the formation of quasi-coherent excitation waves driven by the sizable (as compared to IB) intermolecular electronic 


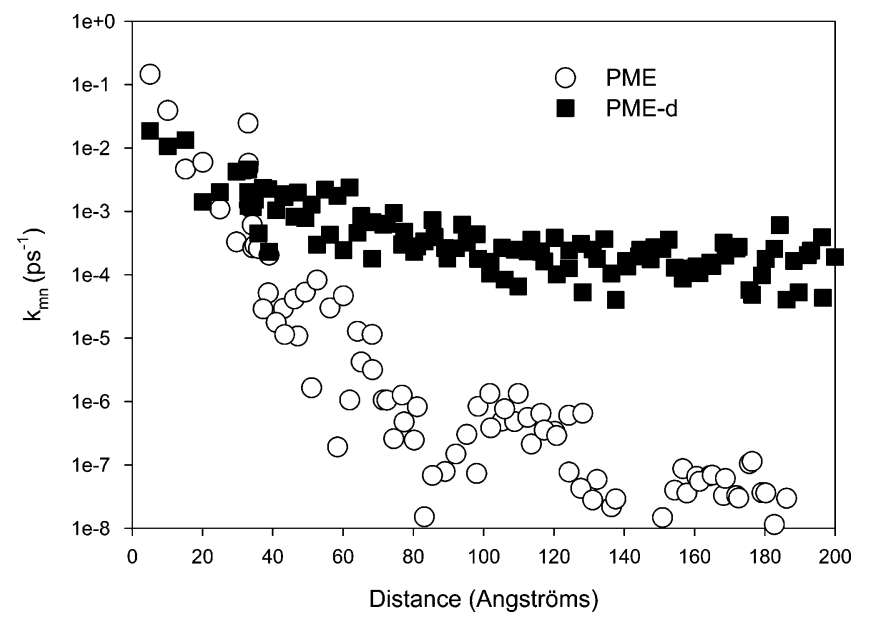

Figure 6. Evolution with donor-acceptor distance of the hopping rate as calculated at the PME and PME-d levels.

couplings. In the limiting case of zero disorder, the aggregates would behave as perfect wires leading to a vanishingly small falloff parameter and coherent transport. It should be stressed, however, that in addition to the inhomogeneity coupling to vibrations is expected to further reduce the exciton coherence length. As this is not fully taken into account here, the PME-d results likely represent an upper limit for the excitation delocalization and the diffusion rates. Yet, it is worth stressing that a better agreement with experiment is obtained when switching from PME to PME-d, suggesting that the electronic excitations spread out over at least several molecules along the stacks. This is also consistent with the very similar timedependent polarization anisotropy decays (Figure 5b) that have been measured for the 3- and 5-unit OPV oligomers, despite the fact that these are likely characterized by different $\beta$ values (smaller $\beta$ values are to be expected for longer segments, due to increased $\pi-\pi$ interactions).

While in the PME case the increase with $\beta$ of the polarization anisotropy drop associated with a single hopping process is not compensated by the increased jump rate (resulting in the strong angle dependence in Figure 5a), the situation is different at the PME-d level; in the range of interchromophore rotation angles considered, the acceptor states display a similar wave function delocalization so that a large and only weakly $\beta$-dependent fraction of the chiral stacks can be explored within a short period of time. That the excited species can sample a larger domain of the disorder-induced DOS in the PME-d model with respect to the PME approach is clearly seen from the calculated rms displacements (Figure 7) and the fate of the electronic excitations as a function of time (Figure 8).

From Figure 7 , we can identify two regimes in the timedependent rms displacements. At early time, $\sqrt{ }\left\langle d^{2}(t)\right\rangle$ evolves strongly with time (within the first $20 \mathrm{ps,} \mathrm{the} \mathrm{excitations} \mathrm{cover}$ $\sim 3$ and $\sim 15 \mathrm{~nm}$, i.e., $\sim 8$ and $\sim 40$ pairs along the stacks at the PME and PME-d levels, respectively); it then slows down for $t$ larger than $\sim 50 \mathrm{ps}$ where $\left\langle d^{2}(t)\right\rangle$ can be reasonably described by a linear dependence (see inset of Figure 7). This illustrates the (progressive) change from dispersive transport at short time delays, where the probability is high for the photoinduced excitation to find chromophores with lower excitation energies resulting in fast downhill migration (consistent with the rapid decay of the mean energy within the first $\sim 50$ ps in Figure 4a), to normal diffusion at longer time delays characterized by a linear scaling of $\left\langle d^{2}(t)\right\rangle$. In this regime, a room temperature diffusion constant of $\sim 5 \times 10^{-4}$ and $\sim 150 \times 10^{-4} \mathrm{~cm}^{2} \mathrm{~s}^{-1}$ is extracted at the PME and PME-d levels, respectively (assuming

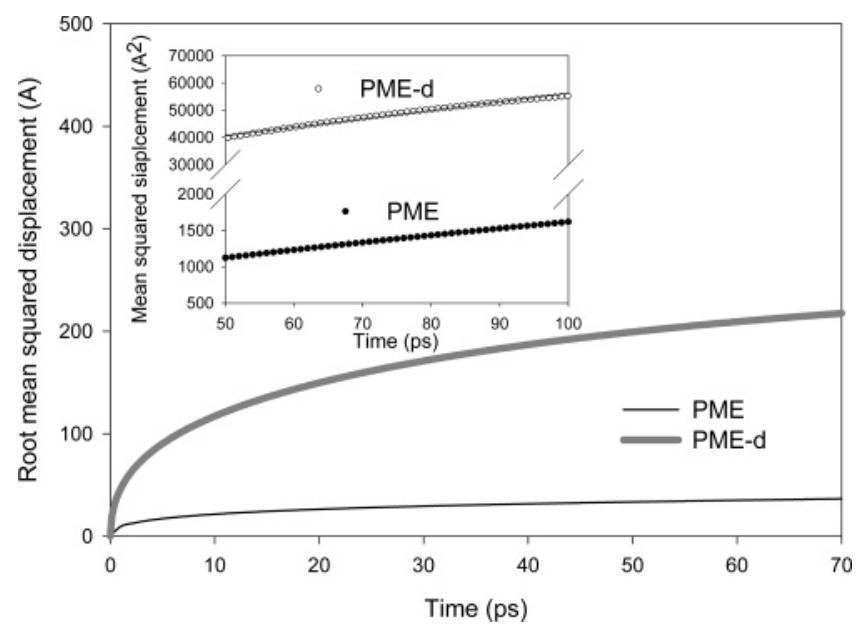

Figure 7. Time-dependent rms displacement in OPV 4 stacks $(\beta=$ $12^{\circ}$ ) as obtained at the PME and PME-d levels. The inset shows the linear dependence with time of the squared displacements for $50 \mathrm{ps}<$ $t<100 \mathrm{ps}$

one-dimensional transport); the fit is based on a time range 50-100 ps (the diffusion constant decreases by less than $20 \%$ when extrapolating the PME results up to $500 \mathrm{ps}$; due to the limited size of the clusters considered here, such extrapolation would be meaningless at the PME-d level).

In the specific simulation displayed in Figure 8, the chromophore labeled 45 is initially excited, and at the PME level, the population is rapidly redistributed among neighbor sites in accordance with their relative energies and the detailed balance conditions. In the PME-d simulations, similar trends are observed, yet a significant fraction of the excitation population reaches OPV4 molecules located further from the absorbing chromophore resulting in a smoother distribution. Interestingly, in both cases, energy can also be transferred efficiently between molecules linked by hydrogen bonds (yet with a smaller probability than along stacks), thereby providing alternative pathways for excitation transport in case migration along the stack direction is unfavored (by reduced spectral overlap).

Next, we have explored the dynamics of energy migration and trapping in mixed stacks of phenylenevinylene oligomers. We have shown previously that highly ordered columnar structures are formed upon cooling dodecane solutions with a few percent OPV4 in OPV3, hence providing an interesting host-guest scaffold for light harvesting. ${ }^{12}$ Figure 9 shows the early time evolution of the relative OPV4 excited-state population, in a stack including $\sim 2.6 \%$ OPV4 guest molecules. As the excitation dynamics have been found to be very similar for the OPV3, OPV4, and OPV5 molecules, we stuck with OPV4 as the host material in the simulations. The presence of longer conjugated oligomers within the stacks has been simply taken into account here by including sites with $\sim 0.2 \mathrm{eV}$ lower excitation energy (corresponding to the difference between OPV3 and OPV4 PL emission peaks) and PL spectra similar to that of the host; these low-energy sites act as traps for the excitations since uphill RET from these chromophores is characterized by much smaller overlaps and rates than the corresponding downhill processes.

The results of these simulations for doping levels of 1.5, 2, and $4 \%$ (and $\beta$ values of $12^{\circ}$ ) are compared to the experimental data in Figure 9. Despite the simplicity of the model used, there is reasonable agreement between experiment and theory, although the simulations tend to overestimate the increase over time of the "trap" population. One possible reason for this is the formation of OPV4 aggregates within the OPV3 stacks 
(a)

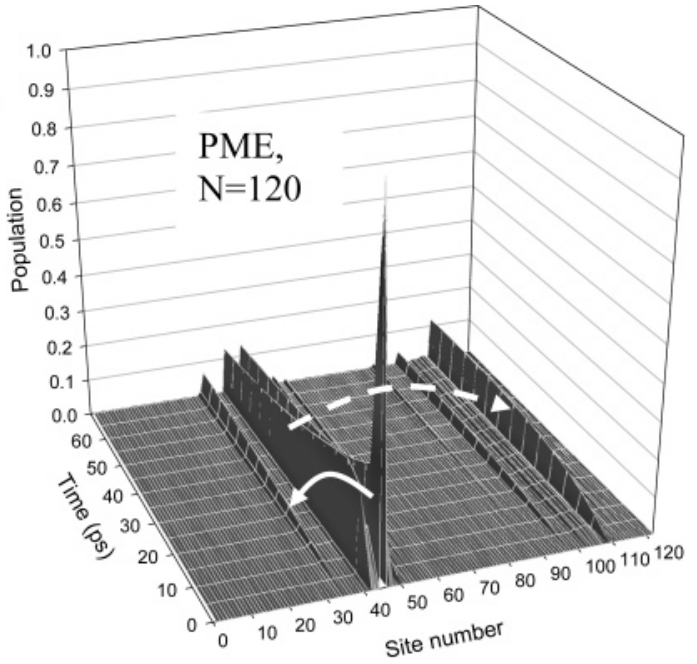

(b)

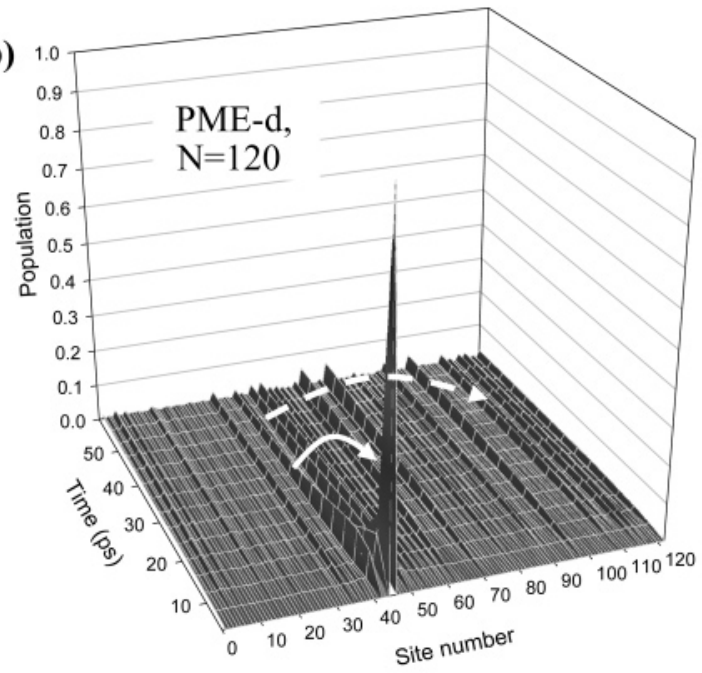

$\mathrm{N} / 2$

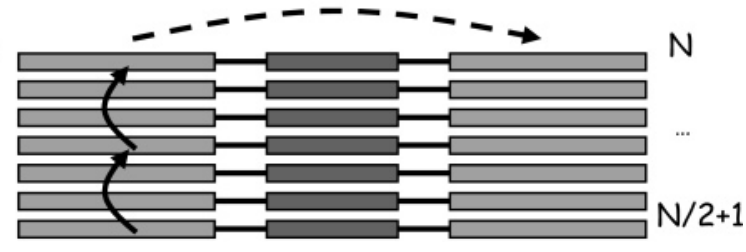

Figure 8. Time-dependent excited-state populations in a stack comprising 120 OPV4 molecules, as predicted at the (a) PME and (b) PME-d levels. The site labeling is shown below. At time zero, the excitation is located on site 45 . Solid and dashed arrows represent motion along the stacking direction and through the hydrogen bridging units, respectively.

(showing up experimentally for doping levels in excess of 2\%), which makes the distinction between host and guest emission more ambiguous. ${ }^{12}$ In contrast to energy diffusion along pure host stacks, the PME and PME-d approximations lead to fairly similar trapping dynamics, at least in the time window we have considered here. In fact, the increase in the trap population at the early stage of the dynamics is mostly controlled by direct Förster energy transfer to the guest molecules of closely lying host chromophores, which explains why it is not sensitive to the diffusion efficiency within the host (at longer time delays, contributions from host molecules excited further away from the traps start playing a role, and a sharper increase in the trap population with time is then predicted at the PME-d level, consistent with the faster transport calculated in the pure host in that case).

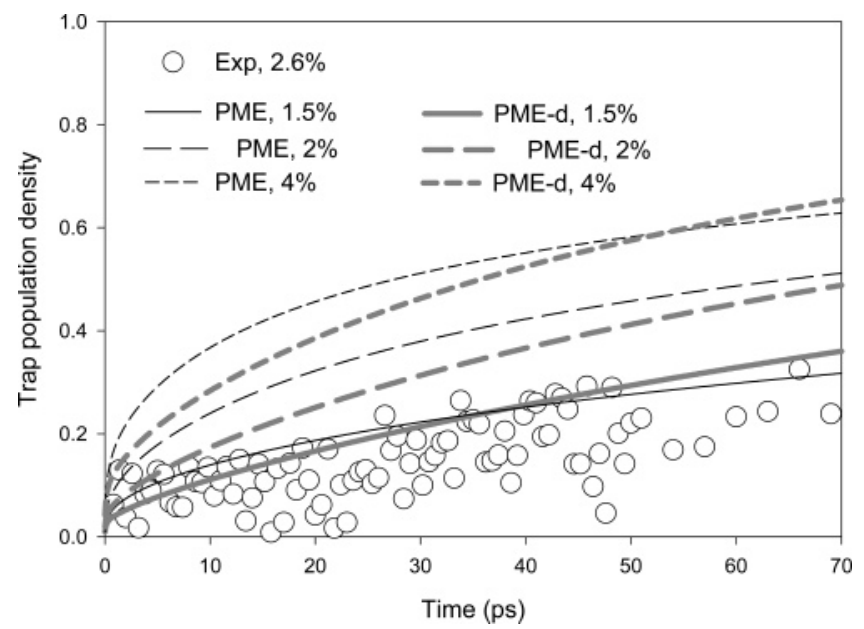

Figure 9. Comparison between the measured (open symbols) and calculated (at the PME and PME-d levels) time-dependent trap populations in guest-host mixed OPV stacks.

Figure 10 pictures one typical simulation of the timedependent chromophore populations for a doping level of $4 \%$, as obtained by solving the PME and PME-d equations (the chromophore labeled 90 is initially excited in the example considered). As described above, the electronic excitation is in both cases rapidly transferred to a few trap sites (two in the specific example) located near the excited chromophore, either by direct Förster energy transfer or after a few hops between host molecules. It is interesting to note that, as in the case of Figure 8 and diffusion along the stacks, the excitations can find their way to traps via the hydrogen bridging units (in the example displayed in Figure 10, one of the two trap sites populated is located on the other branch of the stacked dimer structure). This is further illustrated by the results of the simulations performed when neglecting all interbranch hopping processes, i.e., assuming purely one-dimensional transport (Figure 11). A smaller trap population is indeed predicted in this case.

\section{Conclusions}

The mechanism of energy migration along molecular stacks based on hydrogen-bonded oligophenylenevinylene derivatives has been explored by assessing the validity of different models against time-resolved spectroscopic data. Stacks of a few hundred of molecules are first built on the basis of the input from molecular mechanics calculations. The absorption and emission spectra of the individual molecules are simulated on the basis of a simple two-mode displaced oscillator model with both homogeneous and inhomogeneous contributions to the line widths (adjusted to reproduce the experimental line shapes as well as detailed balance conditions). These are subsequently used to compute spectral overlaps between the simulated homogeneously broadened absorption and emission spectra.

A multicentric monopole expansion based on the results of highly correlated quantum-chemical calculations is applied for the calculation of the electronic couplings between all chromophore pairs. Within the weak coupling regime relevant to incoherent transport, the hopping rates are readily obtained from a FGR expression as the product of the spectral overlaps and the squared electronic couplings between individual chromophores. Injecting the rates computed for each realization of the disorder into a PME formalism and solving these master equations leads to ensemble-averaged time-dependent populations on all sites. The emission polarization anisotropy simulated 
(a)

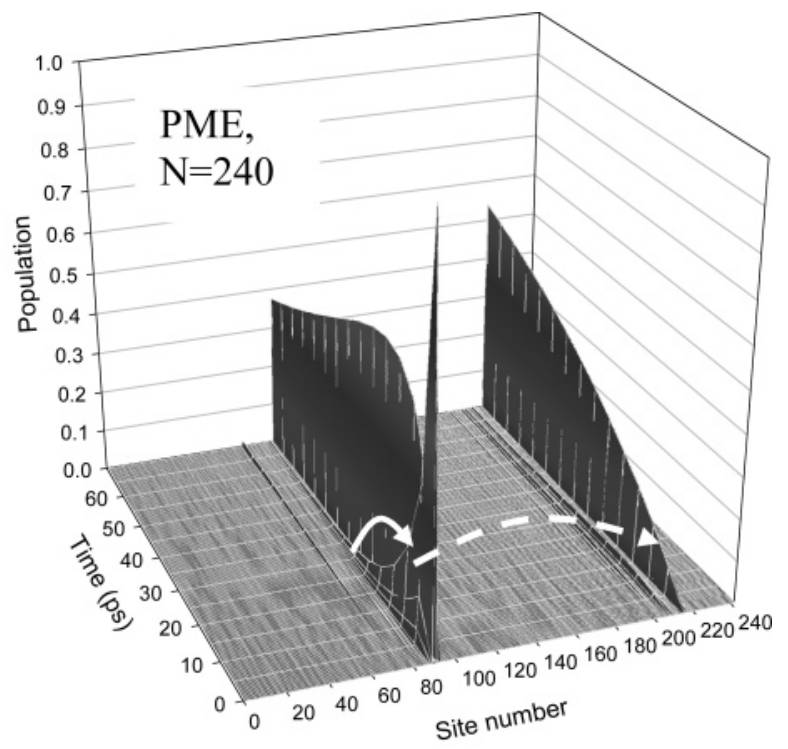

(b)

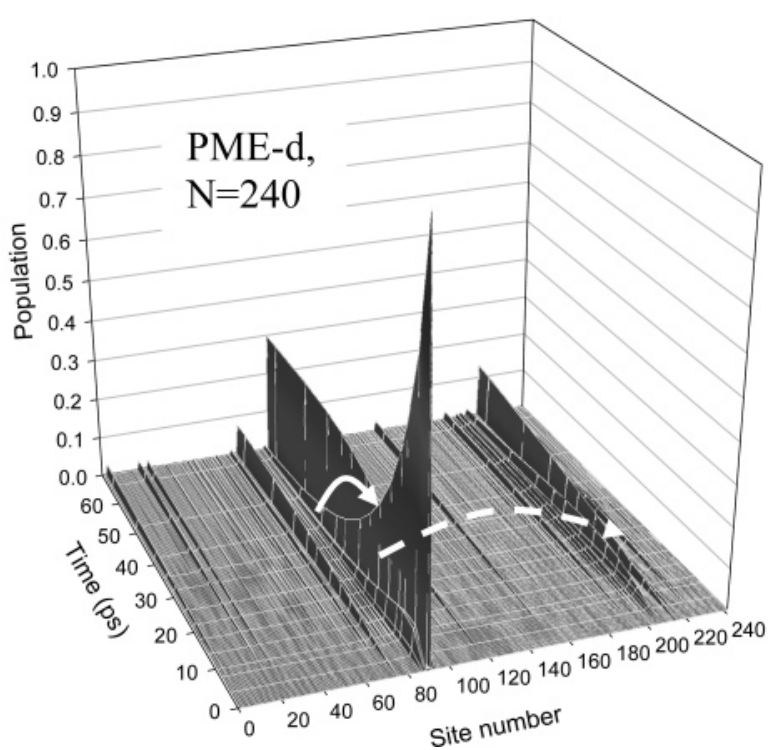

Figure 10. Time-dependent excited-state populations in a stack comprising 240 molecules with $4 \%$ traps, as predicted at the (a) PME and (b) PME-d levels. The site labeling is the same as in Figure 8.

on the basis of the PME populations shows a time decay that is too slow compared to the experimental findings (indicating a complete loss of the initial anisotropy within the first $\sim 30 \mathrm{ps}$ ).

We attribute this discrepancy to the delocalization of the electronic excitations among acceptors (i.e., in the ground-state geometry), which is completely overlooked at the PME level (while geometric relaxation in the excited state, i.e., for donors, very likely leads to rapid self-localization). To account for this effect, we have proposed a modified version of the PME approach (referred to as PME-d for delocalized), where transport occurs from a localized donor state to a set of effective acceptor states built from a superposition of local excitations (as obtained from diagonalization of the acceptor exciton matrix). Despite the crude approximation considered in the calculation of the spectral overlaps involving the acceptor exciton states, the PME-d model leads to a time-dependent polarization anisotropy in much better agreement with experiment. This is rationalized by the formation of quasi-coherent excitation waves leading a much smaller falloff with distance of the hopping rates as compared to the PME simulations. In addition, in contrast to

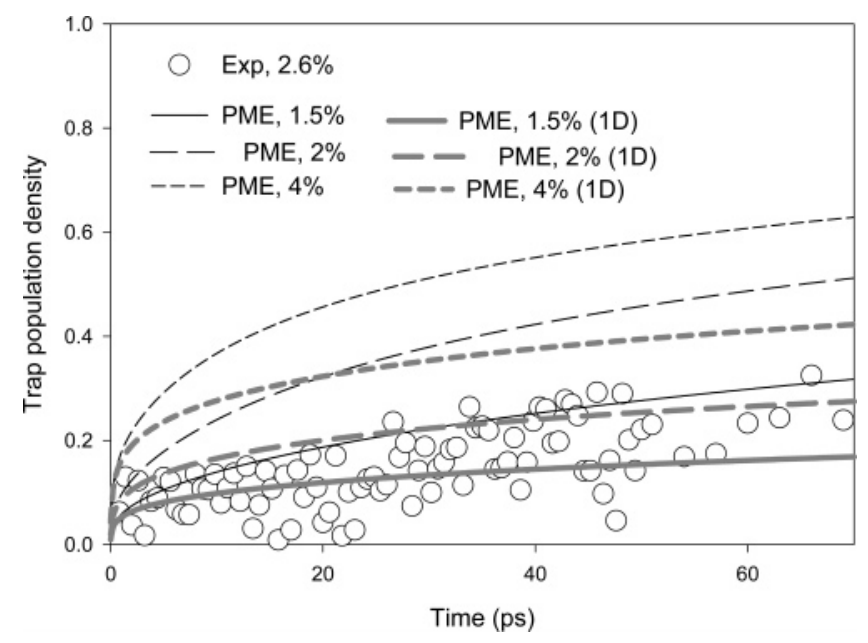

Figure 11. Same as Figure 9 (but only PME simulations) with and without (1D) accounting for hops across the hydrogen-bonded units.

the PME results, the diffusion dynamics predicted at the PME-d level are found to be weakly dependent on the stacking angle $\beta$, which is consistent with the similar polarization anisotropy decays measured for oligomers ranging in size from three to five repeating units (and likely characterized by different $\beta$ values).

The same formalisms were applied to study energy transfer along stacks doped by a few percent of longer oligomers acting as deep traps for the excitations. The early time dynamics are controlled by direct Förster energy transfer from chromophores residing close to the excited molecules; hence the PME and PME-d models lead to a similar buildup with time of the trap population. In both cases, $\sim 20-30 \%$ of the excitations are trapped within the first $20 \mathrm{ps}$ after excitation for a doping level of $2 \%$, in reasonable agreement yet slightly larger than the corresponding experimental value (on the order of 10-20\%). These simulations also suggest that migration paths involving hops across the hydrogen bridges contribute to the overall trapping mechanism, so that with respect to energy transport the chiral stacks should not be viewed as purely one-dimensional objects.

The fast energy migration encountered in the OPV stacks investigated here points to the central role of supramolecular organization on the excitation (and, by extension, charge) dynamics in conjugated materials. It is a nice example illustrating how slight modifications in the chemical (primary) structure of the individual molecules (chiral side chains and hydrogenbonded units) can be used to control their packing and the resulting transport properties. Applying these ideas to tailor the morphology of usually highly disordered conjugated polymers is a formidable challenge to polymer synthesis but would be a real breakthrough in the design of solution processable conjugated materials for applications such as sensors and transistors. On the theoretical side, it also calls for the development of more general yet tractable models that would cover the whole range of interactions, from weak coupling and incoherent transport in disordered materials to strong coupling and coherent motion in well-organized supramolecular or macromolecular architectures. Work is now in progress for extending to conjugated materials the application of these models, which up to now has been mostly limited to the description of light-harvesting phenomena by photosynthetic systems. ${ }^{40}$

Acknowledgment. The work in Mons is partly supported by the Belgian Federal Government "InterUniversity Attraction 
Pole in Supramolecular Chemistry and Catalysis" (PAI 4/11) and the Belgian National Fund for Scientific Research. E. H. and D. B. are a research fellow and associate of the Belgian National Fund for Scientific Research, respectively. G. D. S. thanks the Natural Sciences and Engineering Research Council of Canada for financial support. The work in Cambridge and Oxford is supported by a grant from the U. K. Engineering and Physical Sciences Research Council (EPSRC). C. D. and C. S. thank the IRC Nanotechnology (Cambridge-UCL-Bristol) and the EPSRC (Advanced Research Fellowship), respectively, for financial assistance. The work in Eindhoven is supported by the Royal Netherlands Academy of Arts and Sciences, the Council for Chemical Sciences of The Netherlands Organization for Scientific Research and the Engineering and Physical Science Research Council (U. K.). The Cambridge-Eindhoven-Mons collaboration is supported by the European Commission (LAMINATE) and the European Integrated Project NAIMO (NMP4-CT-2004-500355).

\section{References and Notes}

(1) Burroughes, J. H.; Bradley, D. D. C.; Brown, A. R.; Marks, R. N.; Friend, R. H.; Burn, P. L.; Holmes, A. B. Nature 1990, 347, 539.

(2) Friend, R. H.; Gymer, R. W.; Holmes, A. B.; Burroughes, J. H.; Marks, R. N.; Taliani, C.; Bradley, D. D. C.; dos Santos, D. A.; Brédas, J.-L.; Lögdlund, M.; Salaneck, W. R. Nature 1999, 397, 121.

(3) Sheats, J. R.; Antoniadis, H.; Hueschen, M.; Leonard, W.; Miller, J.; Moon, R.; Roitman, D.; Stocking, A. Science 1996, 273, 884.

(4) Sirringhaus, H.; Brown, P. J.; Friend, R. H.; Nielsen, M. M. Bechgaard, K.; Langeveld-Voss, B. M. W.; Spiering, A. J. H.; Janssen, R. A. J.; Meijer, E. W.; Herwig, P.; de Leeuw, D. M. Nature 1999, 401, 685.

(5) Halls, J. J. M.; Walsh, C. A.; Greenham, N. C.; Marseglia, E. A.; Friend, R. H.; Moratti, S. C.; Holmes, A. B. Nature 1995, 376, 498.

(6) Brabec, C. J.; Sariciftci, N. S.; Hummelen, J. C. Adv. Funct. Mater.

2001, 11,15 .

(7) Schindler, F.; Lupton, J. M. Proc. Natl. Acad. Sci. U.S.A. 2004, 101,14695

(8) Yu, Z. H.; Barbara, P. F. J. Phys. Chem. B 2004, 108, 11321.

(9) Jonkheijm, P.; Hoeben, F. J. M.; Kleppinger, R.; Van Herrikhuyzen, J.; Schenning, A. P. H. J.; Meijer, E. W. J. Am. Chem. Soc. 2003, 125, 15941.

(10) Meskers, S. C. J.; Hübner, J.; Oestreich, M.; Bässler, H. J. Phys. Chem. B 2001, 105, 9139.

(11) Bässler, H.; Schweitzer, B. Acc. Chem. Res. 1999, 32, 173.

(12) Hoeben, F. J. M.; Herz, L. M.; Daniel, C.; Jonkheijm, P.; Schenning, A. P. H. J.; Silva, C.; Meskers, S. C. J.; Beljonne, D.; Phillips, R. T.; Friend, R. H.; Meijer, E. W. Angew. Chem. 2004, 43, 1976. Herz, L. M.; Daniel, C.; Hoeben, F. J. M.; Schenning, A. P. H. J.; Meijer, E. W.; Friend, R. H.; Philipps, R. T. Phys. Rev. B 2003, 68, 045203.
(13) May, V.; Kühn, O. Charge and Energy Transfer Dynamics in Molecular Systems; Wiley: Berlin, 2000.

(14) Kenkre, V. M.; Knox, R. S. Phys. Rev. Lett. 1974, 9, 5279

(15) Schatz, G. C.; Ratner, M. A. Quantum Mechanics in Chemistry; Prentice Hall: Englewood Cliffs, NJ, 1993.

(16) Scholes, G. D. Annu. Rev. Phys. Chem. 2003, 54, 57.

(17) Schenning, A. P. H. J.; Jonkheijm, P.; Peeters, E.; Meijer, E. W. J. Am. Chem. Soc. 2001, 123, 409.

(18) Daniel, C. Ph.D. Thesis, Cambridge, U. K., 2004.

(19) Meskers, S. C. J. et al., to be submitted for publication.

(20) Förster, T. Ann. Phys. 1948, 2, 55. Förster, T. Discuss. Faraday Soc. 1959, 27, 7. Förster, T. Pure Appl. Chem. 1970, 24, 443. Förster, T.; Kasper, K. Z. Elektrochem. 1955, 59, 976.

(21) Marguet, S.; Markovitsi, D.; Millié, P.; Sigal, H.; Kumar, S. J. Phys. Chem. B 1998, 102, 4697.

(22) Beljonne, D.; Cornil, J.; Silbey, R.; Millié, P.; Brédas, J.-L. J. Chem. Phys. 2000, 112, 4749

(23) Krueger, B. P.; Scholes, G. D.; Fleming, G. R. J. Phys. Chem. B 2000, 104, 1854 .

(24) Wiesenhofer, H.; Beljonne, D.; Scholes, G. D.; Hennebicq, E.; Brédas, J.-L.; Zojer, E. Adv. Funct. Mater. 2005, 15, 155.

(25) Spano, F. C.; Siddiqui, S. Chem. Phys. Lett. 1999, 314, 481.

(26) Lax, M. J. Chem. Phys. 1952, 20, 1752. Keil, T. H. Phys. Rev. 1965, 140, 601. Di Bartolo, B.; Powell, R. C. Phonons and Resonances in Solids; Wiley: New York, 1975.

(27) Brédas, J.-L.; Beljonne, D.; Coropceanu, S.; Cornil, J. Chem. Rev 2004, 104, 4971.

(28) Karaburnaliev, S.; Bittner, E.; Baumgarten, M. J. Chem. Phys. 2001, 114,5863 .

(29) Wiesenhofer, H.; Zojer, E.; List, E. J. W.; Scherf, U.; Brédas, J. L.; Beljonne, D. Phys. Rev. Lett., submitted for publication.

(30) Spano, F. et al., to be submitted for publication.

(31) Bacchiocchi, C.; Zannoni, C. Chem. Phys. Lett. 1997, 268, 541

(32) Mayo, S. L.; Olafson, B. D.; Goddard, W. A. J. Phys. Chem. 1990 94, 8897 .

(33) Dewar, M. J. S.; Zoebisch, E. G.; Healy, E. F.; Stewart, J. J. P. J Am. Chem. Soc. 1995, 107, 3702

(34) Beijer, F. H.; Kooijman, H.; Spek, A. L.; Sijbesma, R. P.; Meijer, E. W. Angew. Chem. 1998, 37, 75.

(35) Ridley, J.; Zerner, M. C. Theor. Chim. Acta 1973, 32, 111.

(36) Hirata, S.; Nooijen, M.; Bartlett, R. J. Chem. Phys. Lett. 2000, 326, 255.

(37) Shuai, Z.; Brédas, J. L. Phys. Rev. B 2000, 62, 15452.

(38) Hennebicq, E.; Pourtois, G.; Scholes, G. D.; Herz, L. M.; Russell, D. M.; Silva, C.; Setayesh, S.; Grimsdale, A. C.; Müllen, K.; Brédas, J.-L., Beljonne, D. J. Am. Chem. Soc. 2005, 127, 4744.

(39) Muller, J. G.; Lemmer; U.; Raschke, G.; Anni, M.; Scherf, U.; Lupton, J. M.; Feldmann, J. Phys. Rev. Lett. 2003, 91, 267403.

(40) Heimel, G. Daghofer, M.; Gierschner, J.; List, E. J. W.; Grimsdale, A. C.; Müllen, K.; Beljonne, D.; Brédas, J.-L.; Zojer, E. J. Chem. Phys. 2005, 122, 054501

(41) Jang, S. J.; Newton, M. D.; Silbey, R. J. Phys. Rev. Lett. 2004, 92 218301 